\title{
Verification of prediction for bending rigidity of woven fabric laminated with interlining by adhesive bonding
}

\author{
KyoungOk Kim ${ }^{1}$, Shigeru Inui ${ }^{2}$ and Masayuki Takatera ${ }^{2}$ \\ ${ }^{1}$ Department of Bioscience and Textile Technology, Interdisciplinary Division of Science and Technology, Shinshu \\ University, Japan \\ ${ }^{2}$ Faculty of Textile Science and Technology, Shinshu University, Japan
}

\begin{abstract}
The purpose of this study is to investigate the effect of pressing on bending rigidities of the face fabric, adhesive interlining and bonded composite fabric and verify the prediction method for bending rigidity of those. Predicting methods of bending rigidity for composite with face fabric and adhesive interlining based on laminated theory were verified with measured bending rigidities and thickness of samples. Bending rigidities and thicknesses of woven fabrics, adhesive interlinings and composites with those were measured by KES-FB system. Polytetrafluoroethylene (PTFE) film was used for measuring mechanical properties of pressed adhesive interlining. Bending rigidities of adhesive interlinings became larger and thicknesses of those became thinner than those of before pressing. Bending rigidities of face fabrics didn't change though the thicknesses became thinner than before pressing. It was found that the case of considering mechanical properties of pressed face fabric and pressed interlining was more efficient to predict bending rigidity of composite with laminated model.
\end{abstract}

Keyword: composite, adhesive interlining, mechanical properties, pressing, laminated theory, bending rigidity, woven fabric

\section{Introduction}

Interlining is a layer of fabric inserted between the face and the lining of a garment to give clothing a suitable appearance and stability. Interlining which uses a thermoplastic resin for attaching the face fabric is known as an adhesive or fusible interlining and it is usually used nowadays because of its convenience. Adhesive interlining generally gives a higher level of quality in a garment.

Because of the property changes, adhesive interlining is considered as an important material for clothing and the mechanical properties have been investigated. Several studies about the effects of adhesive interlining were conducted. In1979, Uruma et al. [1] investgated the relationships between the physical properties of textile composite fabric and those constituting face fabric and fusible interlinings experimentally and statistically. In 1987, Okamoto et al. [2] investigated the physical properties and fabric hand of wool blended fabrics interlined with fusible interlinings and compared them to blended fabrics without interlinings by measuring physical properties. In 2003, Matsunashi et al. [3, 4] studied about the behavior of needle penetration in blind stitch sewing and examined in the case where interlining is seemed together with other fabric. In 2007, Jing et al. [5] suggested predicting bond qualities of fabric composites after wash and dry wash based on a principal neural network model. In 1998, Kim et al. [6] investigated the suitability of nonwoven fusible interlinings to thin worsted fabrics with various fabric structural parameters. These studies mainly investigated mechanical properties of different face fabric and different adhesive interlining on the different situation statistically. However studies on pressing effects are still insufficient.

Adhesive interlining is bonded to face fabric by a pressing machine with high heat and pressure. Accordingly, the face fabric and adhesive interlining are pressed at the same time. Thus heat and pressure affect both adhesive interlining and face fabric. Therefore, it is necessary to study changes in mechanical properties of face fabric and adhesive interlining after pressing to understand the properties and effectiveness of adhesive interlining. Thus, the changes of mechanical properties on those, by pressing, were investigated in this study.

On the other hand, bending rigidity was considered as an important property for garment appearance when considering mechanical properties of interlinings. Therefore, it is necessary to predict the bending rigidity of the composite after bonding interlining and there have been some studies about this subject. Shishoo et al. [7] introduced regression equations and investigated the relationship of mechanical properties theoretically and experimentally. Fan et al. $[8,9$ and 10] suggested a set of equations to predict low stress mechanical properties of fused composites from those of composed fabric and fusible interlining fabrics. Jeong et al. [11] reported on the construction of an integrated tool consisting of a neural network to predict mechanical properties. These methods and equations focused on experimental results. Therefore, the relationship between experimental results and theoretical ones still needed to be verified. Kanayama et al. [12, 13] proposed prediction methods about bending rigidity of a composite based on laminate theory for composite structure. These equations were considered useful to predict bending rigidity of that. However, there were still some differences between theoretical values and the predicted ones. Therefore it is more necessary to examine the calculation and measurement method of the parameters for the prediction equations. Furthermore, the studies were conducted in the $70 \mathrm{~s}-80 \mathrm{~s}$ and the making of the adhesive interlining technique was improved following progress of technical skill. Therefore, it is necessary to verify the efficiency of these methods for current adhesive interlining. Thus, Kanayama et al.'s prediction methods were verified and determination of the method for the parameter was supplemented with the measured results in this study. 


\section{Theoretical}
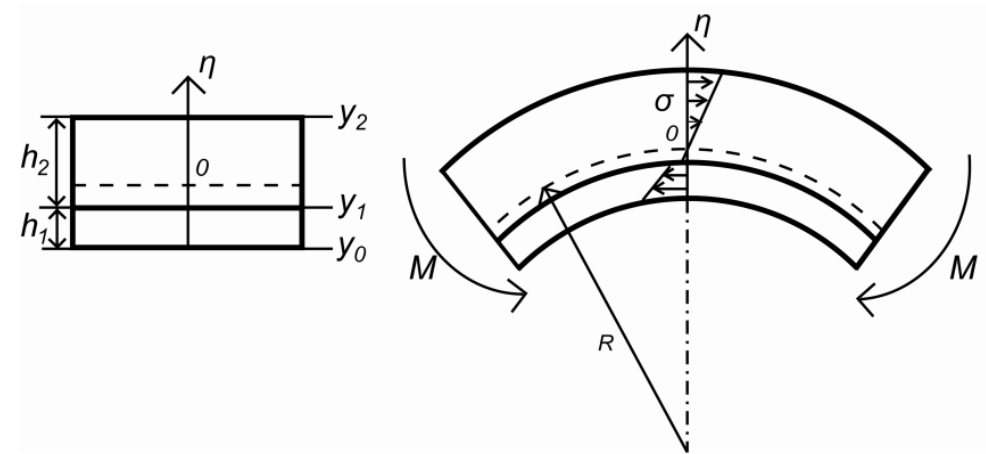

Figure 1 Structure of laminated composite and its bending.

The bending laminated composite of two plates, of which each modulus is different, was considered in this study. The structure of laminated composite is shown in Figure 1. The elastic modulus of each plate is $E_{1}$ and $E_{2}$, and the thickness of each plate is $h_{1}$ and $h_{2}$.

When the laminated composite is bent, the strain distribution in the cross-section is continuous. However the stress distribution is discontinuous at boundary. The neutral surface is not consistent to the symmetry axis of the cross section. In considering bending deformation, strain, $\varepsilon$, is given by

$$
\varepsilon=\frac{\eta}{R}
$$

where $R$ is the radius of curvature for the neutral surface of the composite after bending and $\eta$ is the distance from the neutral surface in a composite. Assuming the Bernoulli-Euler law, the bending moment, $M$, is given by

$$
M=\frac{\overline{E I}}{R}
$$

where $\overline{E I}$ is the equivalent bending rigidity of the composite. From the laminated composite theory of elastic plates, $\overline{E I}$ is given by

$$
\overline{E I}=\frac{b}{3}\left(E_{1}\left[y_{1}^{3}-y_{0}^{3}\right]+E_{2}\left[y_{2}^{3}-y_{1}^{3}\right]\right)
$$

where $y_{0}, y_{1}$ and $y_{2}$, are the coordinates of surface and boundaries from the neutral surface in the cross-section of the laminated plate as shown in Figure 1 and $b$ is the breadth of plates.

In this case, the neutral surface of the composite can be determined by the following relationship.

$$
N=\int_{A} \sigma d A=0
$$

in which $N$ is the resultant force in axial direction of the composite and $\sigma$ is stress.

From Equation 4, we obtain

$$
y_{1}=\frac{h_{1}^{2} E_{1}-h_{2}^{2} E_{2}}{2\left(E_{1} h_{1}+E_{2} h_{2}\right)}
$$

By substituting $y_{0}=y_{1}-h_{1}$ and $y_{2}=h_{2}+y_{1}$ into the Equation 3, we obtain

$$
\overline{E I}=\frac{b}{3}\left(E_{1}\left[h_{1}^{3}+3 y_{1} h_{1}\left(y_{1}-h_{1}\right)\right]+E_{2}\left[h_{2}^{3}+3 y_{1} h_{2}\left(y_{1}+h_{2}\right)\right]\right)
$$

Introducing the moment of inertias, $I$, of each plate,

$$
I_{1}=\frac{b h_{1}^{3}}{12}, I_{2}=\frac{b h_{2}^{3}}{12}
$$

then

$$
\overline{E I}=E_{1}\left[I_{1}+b h_{1}\left(\frac{h_{1}}{2}-y_{1}\right)^{2}\right]+E_{2}\left[I_{2}+b h_{2}\left(\frac{h_{2}}{2}+y_{1}\right)^{2}\right]
$$

Then substituting Equation 5 into Equation 8, after some reductions

$$
\overline{E I}=E_{1} I_{1}+E_{2} I_{2}+3 E_{1} I_{1} E_{2} I_{2} \frac{\left(h_{1}+h_{2}\right)^{2}}{\left(E_{1} I_{1} h_{2}{ }^{2}+E_{2} I_{2} h_{1}^{2}\right)}
$$

In this study, the bending rigidity per unit width of the composite calculated from Equation 9 is denoted by $B_{1}$.

$$
B_{1}=\frac{\overline{E I}}{b}
$$


$E_{1} I_{1}$ and $E_{2} I_{2}$ are the bending rigidity of each plate. They can be measured by a pure bending tester.

The bending rigidity of the composite can be calculated from the bending rigidities and thicknesses for the each plate by using Equation 10. Kanayama et al.[13] used Equation 10 for the prediction of bending rigidity of composites.

Kanayama et al. [14] also proposed an equation considered the effect of adhesive agent with $B_{1}$ as follows.

$$
B_{2}=\left(1+\frac{l_{h}}{l_{s}}\right) B_{1}
$$

where $l_{h}$ and $l_{s}$ are each widths of adhesive agent area and no adhesive resin area of interlining (See Figure 2). They assumed that the shape of adhesive agent area was a rectangle and the adhesive agents were put on regularly following a pattern.
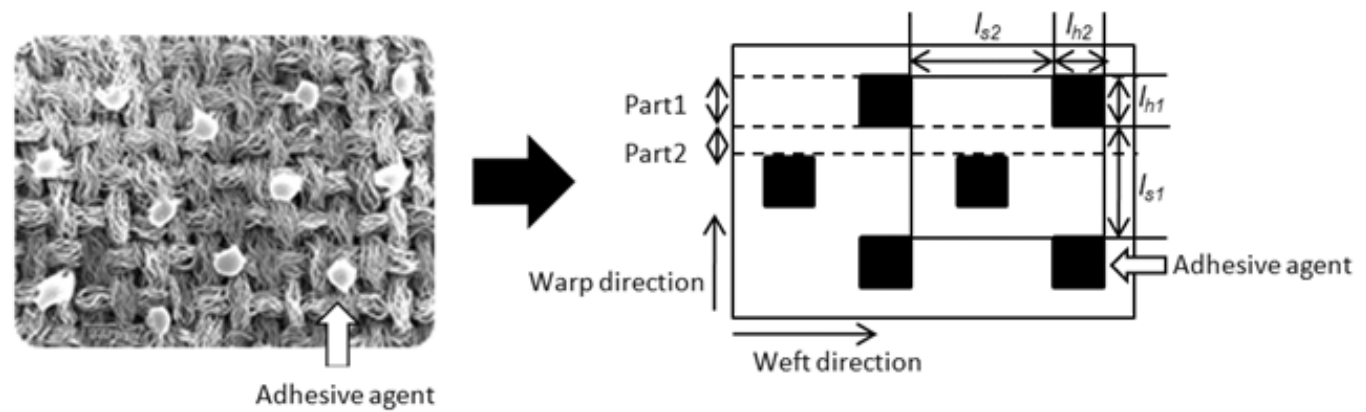

Figure 2 SEM pictures of adhesive interlining (left) and structure model of adhesive interlining from Kanayama et al.(right)

\section{Experimental}

The prediction of the bending rigidity of composite with adhesive interlining with equations 10 and 11 was verified. Face fabrics, adhesive interlinings and the composites were prepared as samples and their bending rigidities and thicknesses were measured and used to verify the equations. The mechanical properties of the face fabric and adhesive interlining may change after the pressing process. Therefore, it was also considered necessary to use the mechanical properties changed by pressing to verify the equations. To measure the mechanical properties changed by pressing, face fabric and adhesive interlining were pressed and the bending rigidity and thickness of each sample was measured and used to verify the equations as well.

Bending properties of each sample were measured by KES-FB2 pure bending tester [14] and the $B$ values of cases where the face fabrics are outside were used. The thickness of each sample was measured by KES-FB3 compression tester at $0.5 \mathrm{gf} / \mathrm{cm}^{2}$ load. Bonding interlining to face fabric was treated by a press machine (KOBE DENKI KOGYOSYO, BP-V4812D) and the bonding conditions were at $150^{\circ} \mathrm{C}$, under $0.3 \mathrm{kgf} / \mathrm{cm}^{2}$ load and for $10 \mathrm{~s}$ pressing time. Every test was carried out under standard conditions (a temperature of $20 \pm 1{ }^{\circ} \mathrm{C}$ and a relative humidity of $65 \pm 5 \%$ ). All samples were treated under standard conditions for 24 hours. Every test was conducted for five samples and the average was used as a result. Changes in cross-section for adhesive interlinings before and after pressing were observed by taking a SEM picture.

Four types of woven fabric made with different yarn count and weave for women's jackets were prepared as face fabrics. Specification of face fabrics and their weave are shown in Table 1. Ten kinds of adhesive interlinings were prepared as samples. Specifications of adhesive interlinings are shown in Table 2 . They were polyester plain fabrics and the adhesive agent was polyamide. The adhesive was double dot which means a structure of the superimposed adhesive dots. Five were controlling density of cloth on weft direction and another five types had a different adhesive agent pattern by controlling the number of adhesive agent dots per area. When the adhesive agent was put on cloth, a screen, which has a thin plate and holes for the adhesive agent, was used. Composites of face fabrics and adhesive interlinings were also prepared as shown in Table 3.

Furthermore, to investigate the pressing effects on the mechanical properties of each sample, bending rigidities and thicknesses of the adhesive interlinings, face fabrics and interlining cloth without adhesive were measured after being pressed individually. Face fabric samples were pressed with the same conditions of bonding interlining and those samples were named as 'pressed face fabric'. The pressing process of adhesive interlining sample was difficult to carry out because the adhesive on adhesive interlining melted when pressed and the adhesive interlining was adhered to the base after pressing. Therefore, polytetrafluoroethylene (PTFE) film (NITTO, No. 900, $0.05 \mathrm{~mm}$ ) was used as a base for pressing the adhesive interlining. Adhesive interlining was bonded to PTFE film by pressing then the PTFE film was removed from the composites as shown in Figure 3. By this process, the adhesive agent can be fixed on the cloths for interlining by pressing to a fabric because the PTFE film is infusible and has a flat surface. Consequently, it was possible to investigate the behavior of the adhesive after pressing. These samples were named as 'pressed interlining'. The properties of cloth for adhesive interlining and the changes of these by pressing were important to understand mechanical properties of adhesive interlining. Therefore, CE-3-NA, which was interlining cloth of CE-3, was prepared as a sample. Those were also pressed and called P-CE-3-NA and P-CE-3 respectively. 


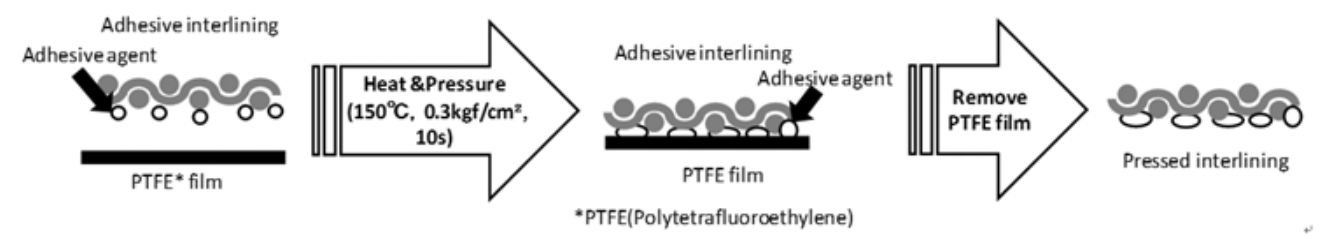

Figure 3 Processing of making pressed interlining.

Table 1 Specification of face fabrics

\begin{tabular}{|c|c|c|c|c|c|c|}
\hline $\begin{array}{c}\text { Sample } \\
\text { name }\end{array}$ & $\begin{array}{c}\text { Yarn } \\
\text { Count(Nm) }\end{array}$ & Weave & Width(cm) & $\begin{array}{c}\text { Density(/inch) } \\
\text { Warp } \times \text { Weft) }\end{array}$ & Material \\
\hline A & $2 / 60 \times 2 / 60$ & Twill & 148 & $72 \times 56$ & Wool $100 \%$ \\
\hline B & $2 / 72 \times 2 / 72$ & Twill & 148 & $74 \times 62$ & Wool $100 \%$ & P-A \\
\hline C & $2 / 72 \times 2 / 72$ & Satin & 148 & $110 \times 74$ & Wool $85 \%$, Angora $15 \%$ & P-B \\
\hline D & $2 / 120 \times 2 / 120$ & Satin & 148 & $132 \times 90$ & Polyester $80 \%$, Wool $15 \%$, Cashmere $5 \%$ & P-D \\
\hline
\end{tabular}

Table 2 Specification of interlining

\begin{tabular}{|c|c|c|c|c|c|c|c|}
\hline $\begin{array}{l}\text { Sample } \\
\text { name }\end{array}$ & $\begin{array}{l}\text { Density } \\
\text { (/inch) }\end{array}$ & $\begin{array}{c}\text { Adhesive } \\
\text { dot number } \\
(/ \text { inch })(\text { warp } \times \text { weft })\end{array}$ & $\begin{array}{c}\text { Adhesive dot } \\
\text { size } \\
(\mathrm{mm})\end{array}$ & $\begin{array}{l}\text { Mass per unit area } \\
\qquad\left(\mathrm{g} / \mathrm{m}^{2}\right)\end{array}$ & $\begin{array}{c}\text { Adhesive } \\
\text { Mass without } \\
\text { Interlining }\left(\mathrm{g} / \mathrm{m}^{2}\right)\end{array}$ & $\begin{array}{c}\text { Screen } \\
\text { thickness } \\
\quad(\mu \mathrm{m})\end{array}$ & Pressed interlining \\
\hline CE-1 & $96.5 \times 55.0$ & $26 \times 26$ & 0.17 & 36.2 & 8.6 & 200 & P-CE-1 \\
\hline CE-2 & $96.0 \times 59.0$ & $26 \times 26$ & 0.17 & 35.6 & 8.0 & 200 & P-CE-2 \\
\hline CE-3 & $95.5 \times 64.0$ & $26 \times 26$ & 0.17 & 36.5 & 8.3 & 200 & P-CE-3 \\
\hline CE-4 & $95.0 \times 66.0$ & $26 \times 26$ & 0.17 & 36.5 & 8.1 & 200 & P-CE-4 \\
\hline CE-5 & $95.0 \times 67.0$ & $26 \times 26$ & 0.17 & 35.7 & 7.7 & 200 & P-CE-5 \\
\hline DP-1 & $98.0 \times 62.0$ & $23 \times 23$ & 0.25 & 38.5 & 8.7 & 200 & P-DP-1 \\
\hline DP-2 & $98.0 \times 62.0$ & $26 \times 26$ & 0.23 & 39.9 & 10.0 & 150 & P-DP-2 \\
\hline DP-3 & $98.0 \times 62.0$ & $26 \times 26$ & 0.30 & 41.8 & 11.6 & 200 & P-DP-3 \\
\hline DP-4 & $98.0 \times 62.0$ & $28 \times 28$ & 0.20 & 37.5 & 8.7 & 200 & P-DP-4 \\
\hline DP-5 & $98.0 \times 62.0$ & $30 \times 30$ & 0.10 & 39.3 & 10.1 & 150 & P-DP-5 \\
\hline
\end{tabular}

Table 3 Specification of composite

\begin{tabular}{|c|c|c|c|c|c|c|c|c|c|c|}
\hline $\begin{array}{r}\text { Adhesive } \\
\text { interlining }\end{array}$ & CE-1 & CE-2 & CE-3 & CE-4 & CE-5 & DP-1 & DP-2 & DP-3 & DP-4 & DP-5 \\
\hline A & A-CE-1 & A-CE-2 & A-CE-3 & A-CE-4 & A-CE-5 & A-DP-1 & A-DP-2 & A-DP-3 & A-DP-4 & A-DP-5 \\
\hline B & B-CE-1 & B-CE-2 & B-CE-3 & B-CE-4 & B-CE-5 & B-DP-1 & B-DP-2 & B-DP-3 & B-DP-4 & B-DP-5 \\
\hline C & C-CE-1 & C-CE-2 & C-CE-3 & C-CE-4 & C-CE-5 & C-DP-1 & C-DP-2 & C-DP-3 & C-DP-4 & C-DP-5 \\
\hline D & D-CE-1 & D-CE-2 & D-CE-3 & D-CE-4 & D-CE-5 & D-DP-1 & D-DP-2 & D-DP-3 & D-DP-4 & D-DP-5 \\
\hline
\end{tabular}

\section{Results and Discussion}

Changes of bending property and thickness for woven fabric and adhesive interlining by pressing

The pressing effects were investigated by comparing the properties of each sample before and after pressing. The thicknesses of face fabrics changed after pressing as shown in Figure 4. However the effects were different for the different weaves. The thicknesses of twill and satin fabric increased and that of plain fabric decreased. It was conceivably due to the effects from heat and pressure during pressing. Therefore, it was found that thicknesses of face fabrics were affected by pressing while bonding adhesive interlining. However, it is necessary to study more about the pressing effects on woven fabric of different weaving.

Even though, the thicknesses of face fabrics were changed by pressing, bending rigidities of pressed face fabric were almost the same as before pressing as shown in Figure 5. Therefore, it was determined that bending rigidities of face fabrics were not affected by pressing.

Thicknesses of pressed interlining were lower than that before pressing as shown in Figure 6. In addition, some changes in the adhesive agent shape were observed after taking SEM pictures as shown in Figure 8. The round shape of the adhesive was flattened and the shape changed. Adhesive agent permeation into space between warp and weft yarns as shown in Figure 9. These changes were also found in all composites. Furthermore, the thicknesses changes of adhesive interlinings and interlining cloth by pressing are shown in Figure 10. Comparing CE-3 to P-CE-3, the thickness of P-CE-3 was lower than that of CE-3. The thicknesses of the adhesive interlinings became clearly thin by pressing. The thickness of CE-3 was lower than the sum of thickness for CE-3-NA and adhesive screen, $200 \mu \mathrm{m}$. The reason was that adhesive agent was permeated into cloth surface during the manufacturing process.

Comparing P-CE-3-NA to CE-3-NA, the thickness of P-CE-3-NA was higher than that of CE-3-NA. With these results, it was conceivable that shrinkage and extension of cloth for adhesive interlining were occurred on adhesive interlining cloth by pressing so the thickness of adhesive interlining changed. Therefore, it is clear that pressing is affected not only adhesive agent but also cloth respectably.

Bending rigidity of pressed interlining increased compared with that before pressing as shown in Figure 7. In addition, bending rigidity of P-CE-3-NA was slightly smaller than that ofCE-3-NA whereas bending rigidity of P-CE-3 increases in comparison with that of CE-3 as shown in Figure 11. Therefore, it is conceivable that the most of pressing process did not affect the bending rigidity of cloth for adhesive interlining and this was similar results with pressed face fabric. With these result, it was concluded that the adhesive agent permeation made adhesive interlining stiffer than before. 


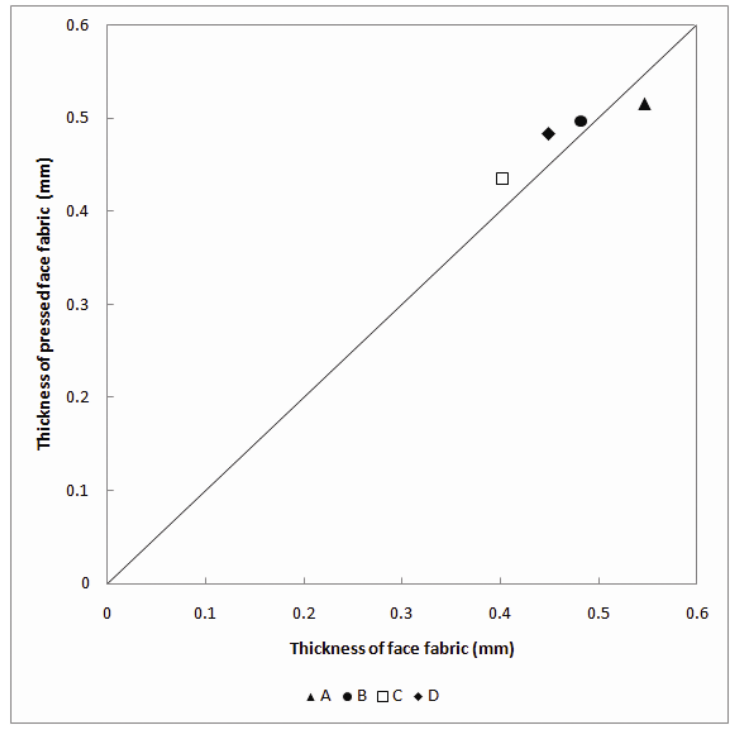

Figure 4 Relationship between thicknesses of pressed face fabrics thickness of face fabrics.

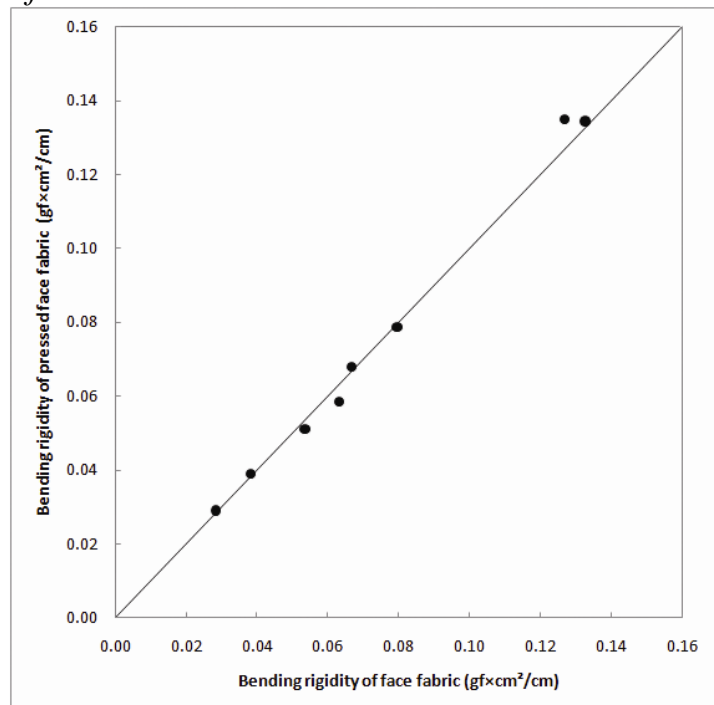

Figure 5 Relationship between bending rigidity of pressed face fabrics and bending rigidity of face fabrics.

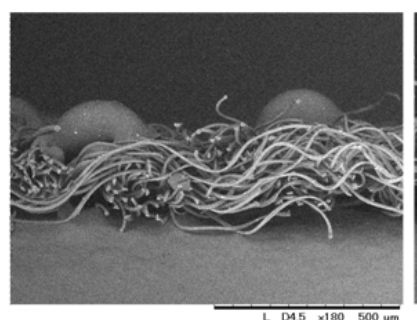

(a)Before pressing(CE3)

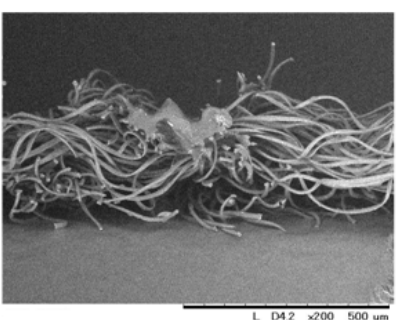

(b) After pressing(CE3)
Figure 8 SEM pictures of Cross section for adhesive interlining: (a) Before pressing and (b) After pressing.

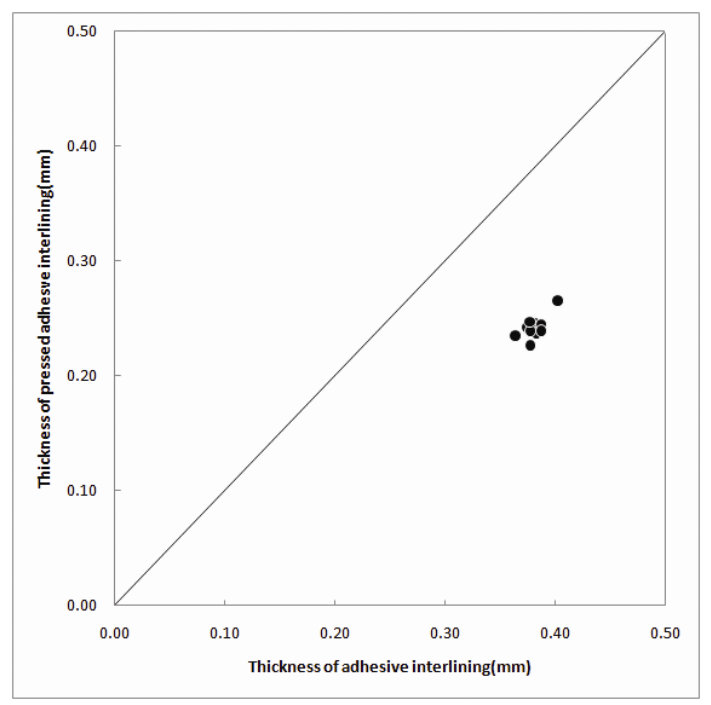

Figure 6 Relationship between thicknesses of pressed interlining and thickness of adhesive interlining before pressing.

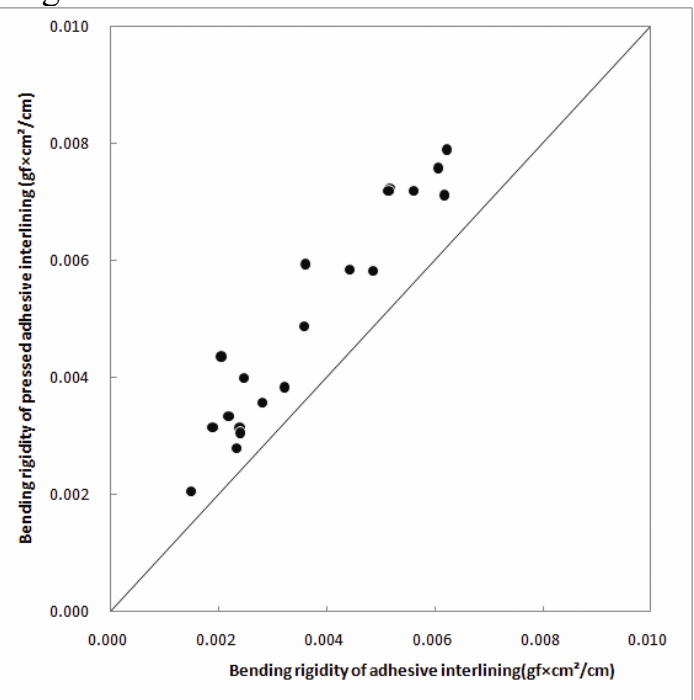

Figure 7 Relationship between bending rigidity of pressed interlining and bending rigidity of adhesive interlining before pressing.
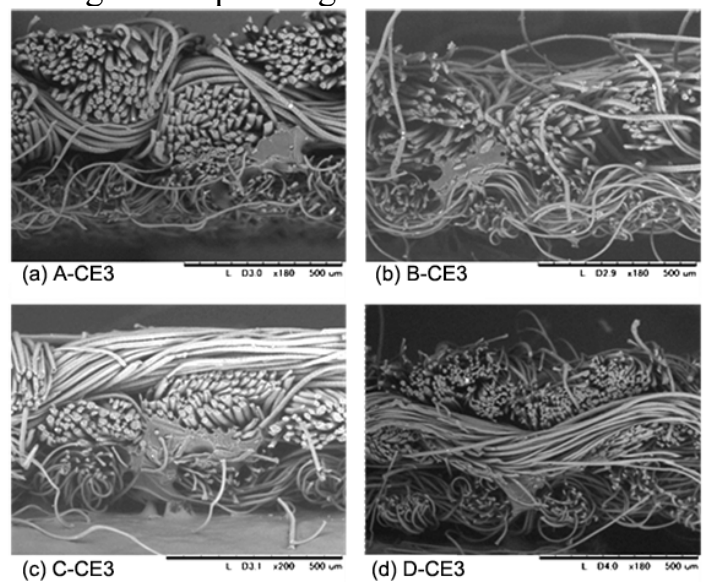

Figure 9 SEM pictures of Cross section for composites: (a) A-CE-3, (b) B-CE-3, (c) C-CE-3, (d) D-CE-3. 


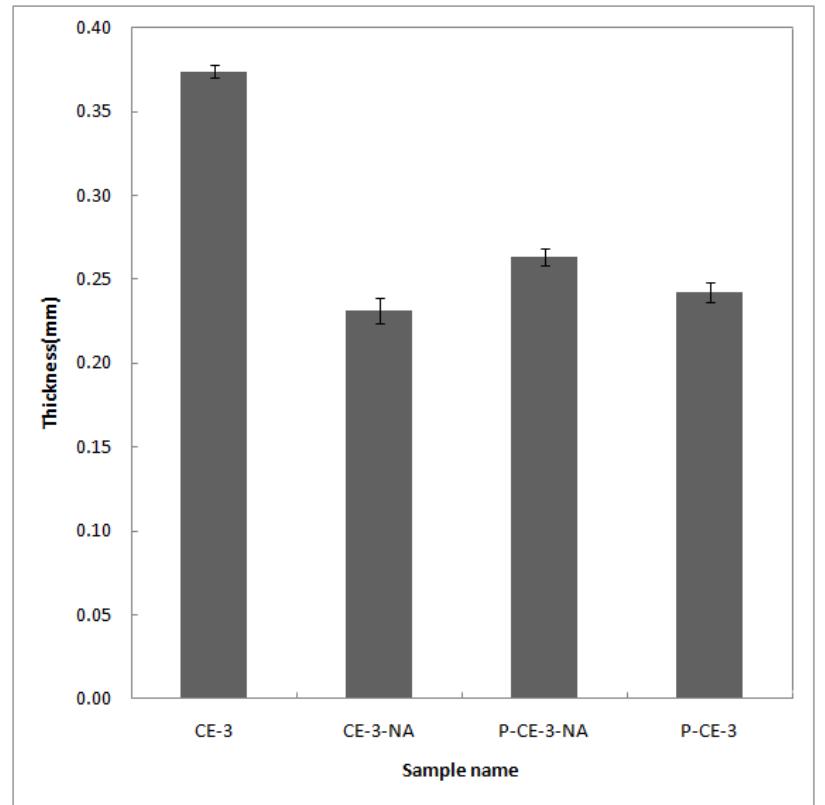

Figure 10 Thickness of adhesive interlining and its cloth without adhesive, before and after pressing.

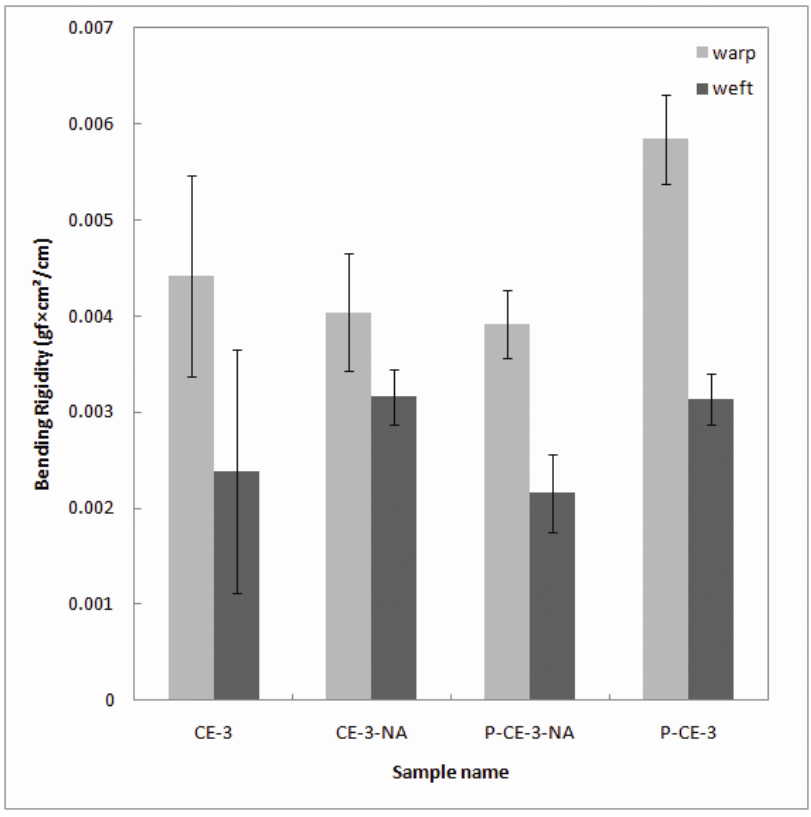

Figure 11 Bending rigidities of adhesive interlining and its cloth without adhesive, before and after pressing.

\section{Prediction of bending rigidity for composite with adhesive interlining}

The Equation 10 and 11 were used to verify with the measured mechanical properties. Bending rigidity and thickness of adhesive interlining before pressing was used to verify Equation 10 and 11 and this case was named as A.I. As previously mentioned, it was found that the pressing process affected the mechanical properties of each sample. Therefore, it was necessary to consider the mechanical properties changes due to pressing. Bending rigidity and thickness of pressed interlining used to verify those equations and that was named as P.I. Furthermore, it was also found that the pressing process affected the mechanical properties of face fabric. Therefore, bending rigidity and thickness of pressed interlining and thickness of pressed fabric was used to verify those equations and named as F.P.I. The list of parameters used for calculation and nomenclature of the result is shown in Table 4.

Comparison of the experimental and calculated bending rigidities with $B_{1}\left(\right.$ A.I.) and $B_{2}$ (A.I.) equations is shown in Figure 12. Comparing results of equation $B_{1}$ (A.I.) to $B_{2}$ (A.I.), $B_{2}$ (A.I.) was slightly closer to the experimental results as a whole. It was because equation $B_{2}$ was considered the adhesive agent effects on $B_{1}$. Comparing the bending rigidity of warp and of weft direction, the lower values of weft direction were agreed with the experimental values better than warp direction. This tendency was also shown in Kanayama's results [12, 13].

The reason why the results from equation $B_{1}$ were not close to experimental ones could be as follows. Firstly, thickness changes of face fabric and adhesive interlining after pressing. Secondly, changes of bending rigidity for adhesive interlining after pressing. In equation $B_{1}$ and $B_{2}$, thicknesses and bending rigidities of samples after bonding were assumed to be the same as that before bonding. However after bonding an adhesive interlining to face fabric, the thickness of the composite after bonding interlinings, become lower than the sum of thickness for face fabric and interlining before bonding. It occurred due to the change of adhesive agent shape and mechanical properties changes of cloth by pressing as mentioned previously. Furthermore, bending rigidity of the adhesive interlining became larger than that before pressing. Therefore it will be necessary to consider those changes to predict the bending rigidity of the composite. In equation $B_{2}$, the percentage of adhesive agent was incorporated to enable the effects of the adhesive agent on bending rigidity to be considered. However, the increasing tendency of bending rigidity with increasing percentage of adhesive agent did not show in the results of $B_{2}$ (A.I.) in Figure 12 . This was because the mass of adhesive agent was not considered in equation $B_{2}$. Some adhesive interlinings had different mass of adhesive agent even though the percentage of those was similar. Therefore not only the percentage of diameter but also the mass will need to be considered when calculating the effect of adhesive agent on bending rigidity of composite.

To consider the changes of mechanical properties for adhesive interlining by pressing, P.I. was used to predict the bending rigidity of the composite. Results of $B_{1}$ (P.I.) and results of $B_{2}$ (P.I.) are shown in Figure 13 . Using bending rigidity values and thickness of pressed interlining, $B_{1}$ (P.I.) was introduced as a simple way to predict bending rigidity by Kanayama et al. However it was necessary to investigate the reason of using the method. In this study, it was found that thickness and bending rigidity of adhesive interlining were changed by pressing. 
Therefore, using P.I. meant that the changes in adhesive interlining by pressing were already considered in the equation. Consequently the results of $B_{1}$ (P.I.) were closer to experimental ones than $B_{1}$ (A.I.) and $B_{2}$ (A.I.). The results of $B_{2}$ (P.I.) were higher than the experimental ones than the results from $B_{1}$ (P.I.). It was not useful to use these properties in $B_{2}$ because the changes from pressing were already considered in the results of P.I. Therefore, equation $B_{1}$ with P.I. would be better to predict a more accurate bending rigidity than $B_{2}$ (P.I.).

However, the face fabric changes by pressing were not considered in the case of P.I. The equations were mainly affected by thickness and bending rigidity changes and also, in this study, it was found that the mechanical properties of face fabric were changed by pressing. Therefore, the thickness change on face fabric by pressing must be considered for predicting the bending rigidity of the composite. To consider the changes of adhesive interlining and face fabric by pressing, F.P.I. was used to predict bending rigidity of composite. The results of $B_{1}$ (F.P.I.) and results of $B_{2}$ (F.P.I.) are shown in Figure 14. Root mean square of errors (RMSE) and coefficient of determination of experimental results and those of each condition are shown in Table 5. RMSE of $B_{1}$ (F.P.I.) was lower than that of $B_{1}$ (P.I.). Therefore, it was found that the results considered thickness changes after pressing face fabric gave a more exact prediction of experimental ones. Consequently, it will be possible to predict bending rigidity of the composite with adhesive interlining with equation $B_{1}$ under F.P.I. conditions more precisely.

Furthermore, Kanayama et al. [12,13] calculated 3 types of face fabric (warp knit, plain woven and nonwoven). In their results, results of nonwoven agreed with experimental ones. The results from the plain woven showed larger values than the experimental ones. However, the results of the woven fabric, which was used in this study, showed closer values to the experimental ones. This could be because of the technical improvement in manufacturing the adhesive interlining. The interlining cloth was getting thinner and adhesive agent mass was getting smaller than the samples from Kanayama et al. Efficiency improvement of the adhesive interlining reduced the space between face fabric and adhesive interlining after bonding. It made the error decrease so that it was possible to predict the bending rigidity of composites.

Table 4 Parameters used for calculation and nomenclature

\begin{tabular}{|c|c|}
\hline Symbol & Condition \\
\hline A.I. & Used bending rigidity and thickness of adhesive interlining before pressing \\
\hline P.I. & Used bending rigidity and thickness of pressed interlining \\
\hline F.P.I. & Used bending rigidity and thickness of pressed interlining and thickness of pressed fabric \\
\hline
\end{tabular}

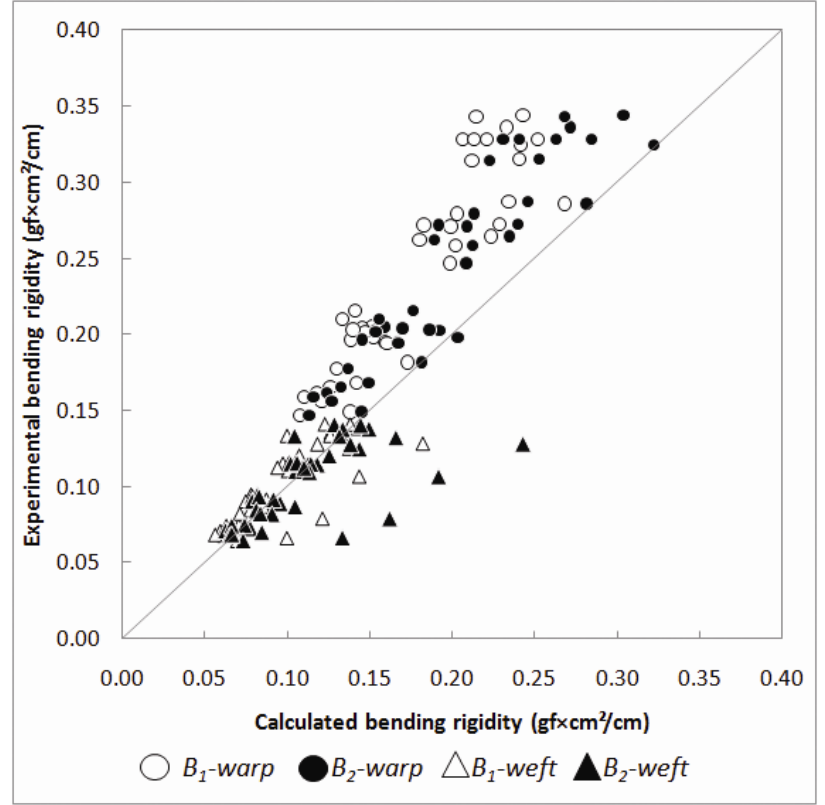

Figure 12 Comparison between experimental bending rigidities and theoretical bending rigidities using $B_{1}$ (A.I.) and $B_{2}$ (A.I.)

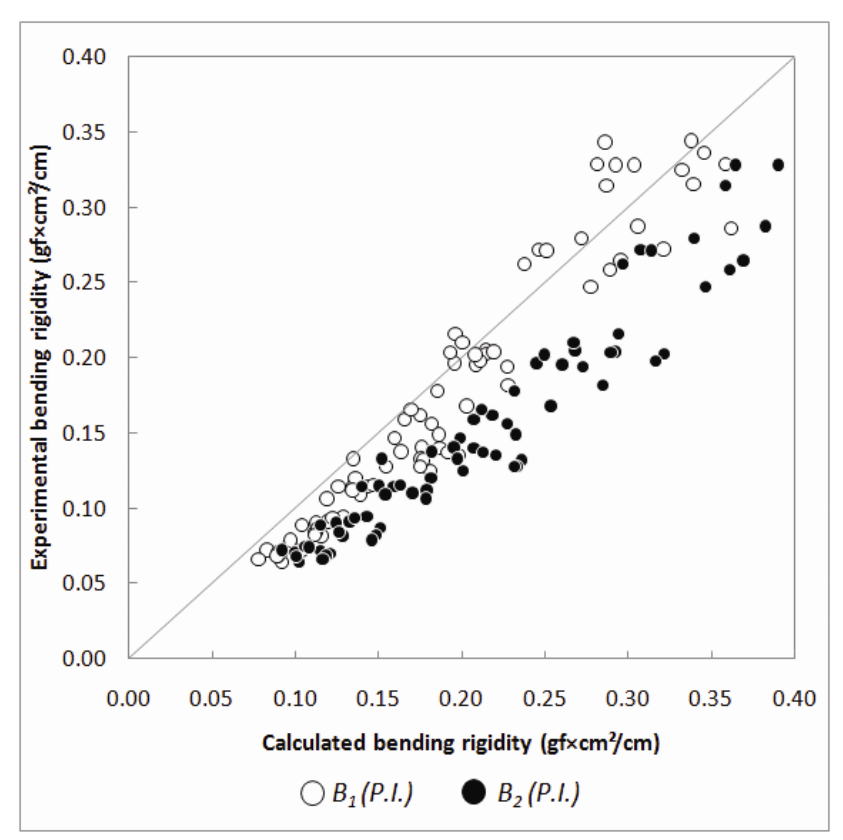

Figure 13 Comparison between experimental bending rigidities and theoretical bending rigidities using $B_{1}$ (P.I.) and $B_{2}$ (P.I.). 


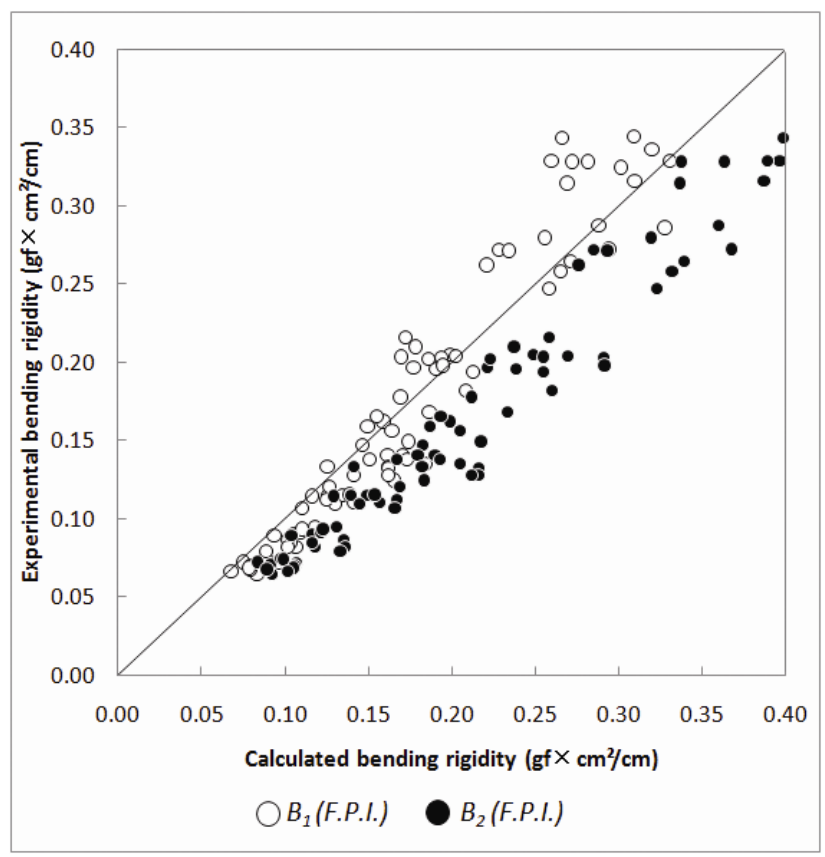

Figure 14 Comparison between experimental bending rigidities and theoretical bending rigidities using $B_{1}$ (F.P.I.) and $B_{2}$ (F.P.I.).

Table 5 Root mean square of errors (RMSE) and coefficient of determination $\left(\mathrm{R}^{2}\right)$ between experimental results and those of each condition

\begin{tabular}{|c|l|c|}
\hline Conditions & RMSE & $\mathrm{R}^{2}$ \\
\hline$B_{1}$ (A.I.) results and experimental results & 0.0056 & 0.8817 \\
\hline$B_{2}$ (A.I.) results and experimental results & 0.0046 & 0.8325 \\
\hline$B_{1}$ (P.I.) results and experimental results & 0.0039 & 0.9263 \\
\hline$B_{2}$ (P.I.) results and experimental results & 0.0085 & 0.9355 \\
\hline$B_{1}$ (F.P.I.) results and experimental results & 0.0029 & 0.9264 \\
\hline$B_{2}$ (F.P.I.) results and experimental results & 0.0062 & 0.9405 \\
\hline
\end{tabular}

\section{Conclusions}

The changes of mechanical properties, for adhesive interlining, face fabric and composites of these, by pressing were investigated and the predicting methods were verified with measured data. It was found that not only the properties of adhesive interlining but also the properties of face fabric changed in the pressing process. It was also found that the pressing process had also effects on cloth for adhesive interlining. The predicting methods for bending rigidity of composite with adhesive interlining and face fabric suggested by Kanayama et al. based on laminate theory for the laminated composite were verified. Comparing results of $B_{1}$ and those of $B_{2}$, those of $B_{2}$ which considered the adhesive agent area were closer to experimental results in the case of using mechanical properties of samples before pressing. However $B_{1}$ was closer to the experimental results in the case of results considering the pressing effects, which used bending rigidity and thickness of pressed interlining with $B_{1}$. Furthermore, the case of considering thickness of the pressed face fabric was more efficient at predicting bending rigidity of composite with $B_{1}$. With these results, it was concluded that the Equation 10 was useful to predict bending rigidity of composites with adhesive interlining, with mechanical properties, the pressing effects on adhesive interlining and face fabric were considered.

The entire predicted results for bending rigidities from this method agreed with experimental ones. For the prediction having higher accuracy, improvement of the further model will be necessary. Therefore, future studies will need to address this. 


\section{Acknowledgements}

We express our appreciation to NITTOBOSEKI CO.,LTD for providing the experimental samples. This work was supported by Grant-in-Aid for Global COE Program by the Ministry of Education, Culture, Sports, Science, and Technology. This paper is dedicated to the first principal, Chotaro Harizuka, on the occasion of 100th anniversary of Faculty of Textile Science and Technology, Shinshu University.

\section{References}

1. Masao Uruma and Mio Tomimori, Relations between the physical properties of a textile composites fabric and its component fabric, SEN'I GAKKAISHI, Vol.35, No.8, Tp357-360 (1979)

2. Yoko Okamoto et al., Changes of hand wool/ polyester blend fabrics for men's suits by interlinings and changes of their physical properties by cleaning, Journal of the Japan Research Association for textile end uses, 19, pp.466-471, (1987)

3. Kuniko Matsunashi and Kozo Shimazaki, Influence of sewing conditions on the amount of needle penetration in two-ply fabrics(interlining and outer fabric) using blind-stitch sewing machine, SEN'I GAKKAISHI, 59(12), 492-498 (2003)

4. Kuniko Matsunashi and Kozo Shimazaki, Simulation of sewing in lapel part using blind-stitch sewing machine and a relation between sewing factors and configuration of lapel, SEN'I GAKKAISHI, 60(8), 245-251 (2004)

5. Jing Wang and Weiyuan Zhang, Predicting bond qualities of fabric composites after wash and dry wash based on principal neural network model, Textile Research Journal, 77(3), 142-150, (2007)

6. S. J. Kim et al., Suitability of nonwoven fusible interlining to the thin worsted fabrics, International Journal of clothing Science and Technology, 10(3/4), 273-282 (1998)

7. Roshan Shishoo, Per Henrik Klevermar, Margareta Cednas, and Bertul Olofsson, Multilayer Textile Structure, Textile Research Journal, 669-679 (1971)

8. J. Fan, W. Leeuwner, and L. Hunter, Compatibility of Outer and Fusible Interlining Fabrics in Tailored Garments, part 1: Desirable range of mechanical properties of fused composites, Textile Research Journal, 67(2), 137-142 (1997)

9. J. Fan, W. Leeuwner, and L. Hunter, Compatibility of Outer and Fusible Interlining Fabrics in Tailored Garments part 2: Relationship between mechanical properties of fused composites and those of outer and fusible interlining fabrics, Textile Research Journal, 67(3), 194-197 (1997)

10. J. Fan, W. Leeuwner, and L. Hunter, Compatibility of Outer and Fusible Interlining Fabrics in Tailored Garments part 2: Selecting fusible interlinings, Textile Research Journal, Vol. 67, No. 4, pp. 258-262 (1997)

11. Sung H. Jeong, Jung H. Kim and Cheol J. Hong, Selecting Optical Interlinings with a neural Network, Textile Research Journal, 70(11), pp.1005-1010, (2000)

12. Machiko Kanayama and Masako Niwa, Bending Properties of Composite Fabrics Reinforced with Fusible Interlinings, Journal of Textile Machinery society of Japan, Vol. 35(1), pp. 102-112 (1981)

13. Machiko Kanayama and Masako Niwa, Theoretical calculation of bending rigidity of fused fabric composite, Journal of Textile Machinery society of Japan, 37(10), pp. T161-T167, (1984)

14. S. Kawabata, The standardization and analysis of hand evaluation 2nd ed., Textile Machinery society of Japan (1980) 